\title{
Cost-effective storage solution for delivering umbilical cord with efficient isolation of mesenchymal stem cells
}

\author{
Baoyu Lai ${ }^{\ddagger}, 1$, Jieyong Huang ${ }^{\ddagger}, 1$, Jieting Chen ${ }^{\ddagger}, 2$, Wenlei Li ${ }^{\ddagger}, 3$, Huimei Wu ${ }^{1}$ \& Jianyong Xu*,1 \\ ${ }^{1}$ Department of Immunology, Health Science Center, Shenzhen University, Shenzhen 518055, PR China; ${ }^{2}$ Department of Obstetrics, People's Hospital of Baoan, Shen- \\ zhen 518045, PR China; ${ }^{3}$ Department of Obstetrics, Women \& Children Health Institute of Futian, Shenzhen 519045, PR China; *Author for correspondence: xujiany- \\ ong@szu.edu.cn; ${ }^{\ddagger}$ Authors contributed equally
}

BioTechniques 68: 53-56 (July 2020) 10.2144/btn-2019-0146

First draft submitted: 5 November 2019; Accepted for publication: 27 February 2020; Published online: 14 April 2020

\section{ABSTRACT}

Mesenchymal stem cells (MSCs) represent a promising therapeutic candidate for treating many diseases. However, their proliferation and therapeutic abilities decline during the aging process and disease development. Therefore, fetal MSCs derived from the umbilical cord (UC) attract more attention. Storing and delivering the UC is one critical step for efficient MSC isolation. Although the culture medium-based solution is suitable for UC storage, it is not feasible for large-scale preparation because of its high price. Thus, we demonstrate here that a simple solution containing a $\mathrm{pH}$ buffering reagent, calcium, magnesium and glucose could be used as a cost-effective storage solution for UC delivery and efficient MSC isolation.

\section{METHOD SUMMARY}

The human umbilical cord was cut off within 5-10 min after the baby's birth, stored in a simple solution containing a pH buffering reagent, calcium, magnesium and glucose plus antibiotics on ice, and then delivered to the laboratory. Mesenchymal stem cells were isolated with collagenase $\mathrm{B}$ digestion after mincing the umbilical cord into approximately $3 \mathrm{~mm}^{3}$ fragments.

KEYWORDS:

delivering solution • mesenchymal stem cells • storage solution $\bullet$ umbilical cord

Mesenchymal stem cells (MSCs) represent a promising therapeutic candidate for treating many diseases, such as graft versus host diseases [1], cardiovascular diseases [2], diabetes [3] and autoimmune diseases [4-6]. MSCs have both multi-potency and immune modulation capabilities, leading to microenvironment refinement and tissue regeneration [7-12].

MSCs could be isolated from multiple tissues, such as bone marrow, adipose and umbilical cord (UC) [12]. However, their proliferation and therapeutic abilities decline during the aging process and disease development [6]. Thus, fetal MSCs derived from UC (UC-MSCs) [13], composed of embryonic mucous connective tissues [13,14], have attracted more attention during the past decades [13]. Indeed, UCMSCs have a faster doubling time, higher colony-forming efficiency and greater expansion capacity with longer telomeres than adult MSCs [13,15-19]. Furthermore, as normally discarded after birth, the UC is easily accessible without requiring an invasive procedure and ethical concerns [14,20]. Given these advantages, UC-MSCs are becoming an alternative source of MSCs for preclinical studies and clinical applications to bone marrow-derived MSCs.

However, the standard procedures for collecting, storing and delivering UCs have not been fully developed and demonstrated. Thus, we conducted the present study to develop a cost-effective storage solution for delivering UCs with efficient isolation of MSCs. Our data indicate that a simple solution containing a pH buffering reagent, calcium, magnesium and glucose, referred to as CMGL solution, could be used as a cost-effective storage solution for UC delivery and efficient MSC isolation.

\section{Human umbilical cord collection \& delivery}

This study was approved by the ethics committee of Shenzhen University (China) and followed the tenets of the Declaration of Helsinki. Informed consent was obtained from all participants. The human UC was cut off within 5-10 min after the baby's birth and stored in the indicated storage solution plus antibiotics (500 units/ml penicillin and $500 \mu \mathrm{g} / \mathrm{ml}$ streptomycin; Thermo Fisher Scientific, MA, USA) on ice and delivered to the laboratory.

\section{Human MSC isolation \& expansion}

The UC was minced into approximately $3 \mathrm{~mm}^{3}$ fragments and stored in the storage solution for the indicated time. Then, it was incubated in $1 \mathrm{mg} / \mathrm{ml}$ collagenase B (STEMCELL Technologies, BC, Canada) for $12 \mathrm{~h}$ at $37^{\circ} \mathrm{C}$. The fragments were washed with phosphate- 
(A)

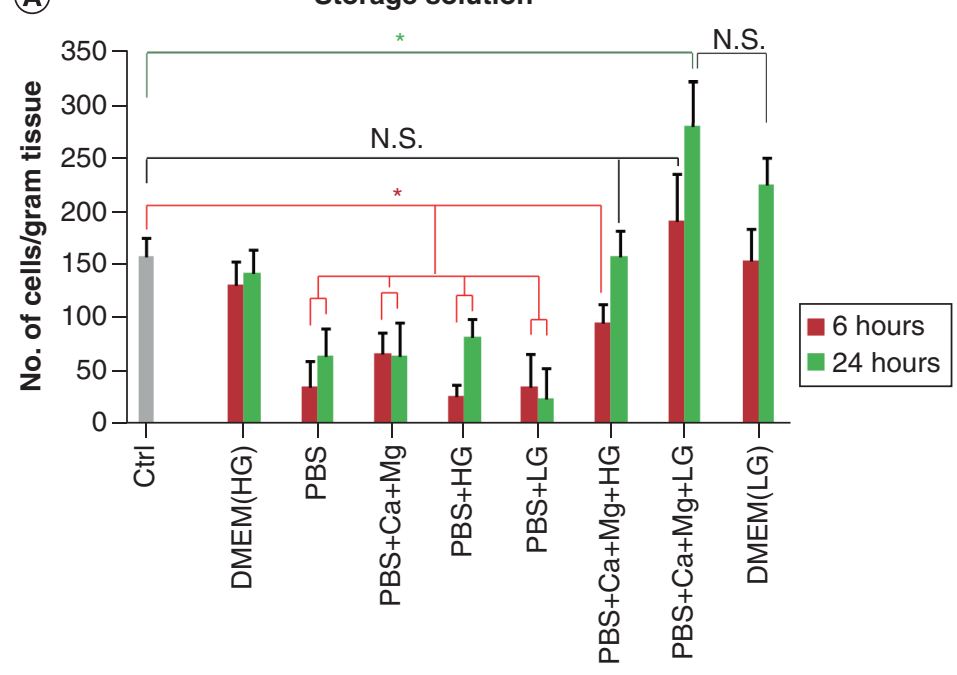

(B)
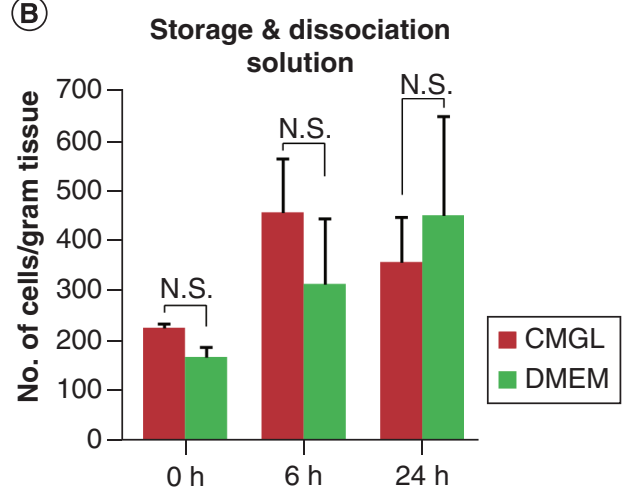

Figure 1. Development of cost-effective storage solution for delivering umbilical cord with efficient isolation of mesenchymal stem cells. The cell number was counted 1 week after the cell plating. The MSCs isolation efficiency was assessed by dividing the counted cell number by the weight of the UC for cell isolation. The experiment was conducted in triplicate. (A) The UC was stored in the indicated solution at $4{ }^{\circ} \mathrm{C}$ for 6 or $24 \mathrm{~h}$. The MSCs isolated immediately from the UC after the birth $(<30 \mathrm{~min})$ without storing in any of the solutions were used as positive controls (Ctrl). (B) The UC was stored in the DMEM or CMGL at 4 C for 6 or $24 \mathrm{~h}$, and then the MSCs were dissociated with collagenase B diluted with DMEM or CMGL. The MSCs isolated immediately from the UC after the birth ( $<30 \mathrm{~min}$ ), without storing in any of the solutions and with collagenase B diluted with DMEM or CMGL, were used as positive controls $(0 \mathrm{~h})$.

* $\mathrm{p}<0.05$.

Red *: Decreased efficiency compared with controls.

Green *: Increased efficiency compared with controls.

CMGL: Our newly developed cost-effective storage solution containing calcium, magnesium, glucose-low concentration in PBS; DMEM: DMEM/high glucose; HG: High glucose (4.5 g/l); LG: Low glucose (1 g/l); MSC: Mesenchymal stem cell; NS: No significance; UC: Umbilical cord.

buffered saline (PBS), plated onto p12 culture plates in DMEM/high glucose plus 10\% fetal bovine serum for 3 days. The fragments were then washed with PBS and replated one more time. The unattached fragments were discarded and the isolated cells were expanded in MSC expansion medium (Dulbecco's Modified Eagle's medium [DMEM]/High Glucose plus 10\% fetal bovine serum, $10 \mathrm{ng} / \mathrm{ml}$ bFGF) (Peprotech, London, UK), $50 \mu \mathrm{g} / \mathrm{ml}$ vitamin C (Sigma, MO, USA), $100 \mathrm{units} / \mathrm{ml}$ penicillin and $100 \mu \mathrm{g} / \mathrm{ml}$ streptomycin [5,21].

\section{Human MSC characterization \& differentiation}

Human MSCs were passaged with TrypLE (ThermoFisher Scientific) for three passages, and then they were subjected to cell surface marker analysis and target differentiation. Differentiation and characterization were performed as described previously [5,21]. Briefly, differentiation was performed with the StemPro ${ }^{\circledR}$ Chondro-genesis Differentiation Kit (Gibco, MA, USA), the StemPro Adipogenesis Differentiation Kit (Gibco) and the StemPro Osteogenesis Differentiation Kit (Gibco) according to manufacturer instructions. The cells were differentiated for 28 days and stained with alcian blue for chondrocyte differentiation, oil red $\mathrm{O}$ for adipocyte differentiation and alizarin red $\mathrm{S}$ for osteocyte differentiation.

\section{Statistical analysis}

Data were analyzed using SPSS software for Windows (SPSS Inc., IL, USA) and are shown as mean \pm standard error of the mean. Student t-test was used for two-group comparison and one-way analysis of variance for multiple group comparison with normal data distribution, parametric test and Turkey post hoc tests. $\mathrm{p}<0.05$ was considered statistically significant.

Although storage solutions for delivering human UC have not been demonstrated in detail, DMEM/high glucose plus antibiotics is the storage solution used most in our laboratory [21], which supports efficient MSC isolation up to $48 \mathrm{~h}$ after storing at $4^{\circ} \mathrm{C}$ or on ice. However, the complicated formulation of DMEM makes it neither cost nor labor effective for large-scale manufacturing. Therefore, it is necessary to develop one simple storage solution for UC delivery with efficient MSC isolation. Theoretically, several basic factors are required, including the $\mathrm{pH}$ buffering system, osmotic pressure maintenance, basic nutrients, co-factors for cell attachment and migration. Therefore, $\mathrm{PBS}$ was used to maintain the $\mathrm{pH}$ and osmotic pressure; glucose was chosen for the basic nutrient; and calcium and magnesium were selected as the co-factors for cell attachment and migration.

To test whether the combination of glucose, calcium and magnesium in PBS could support efficient MSC isolation after storing the $\mathrm{UC}$ for a short (6 $\mathrm{h}$ ) or long period ( $24 \mathrm{~h}$ ), their different combinations were compared (Figure 1A \& Table 1). Data showed that the solution containing a low concentration of glucose $(1 \mathrm{~g} / \mathrm{l})$ plus calcium and magnesium in PBS, as the CMGL, was the most efficient solution 
(A)
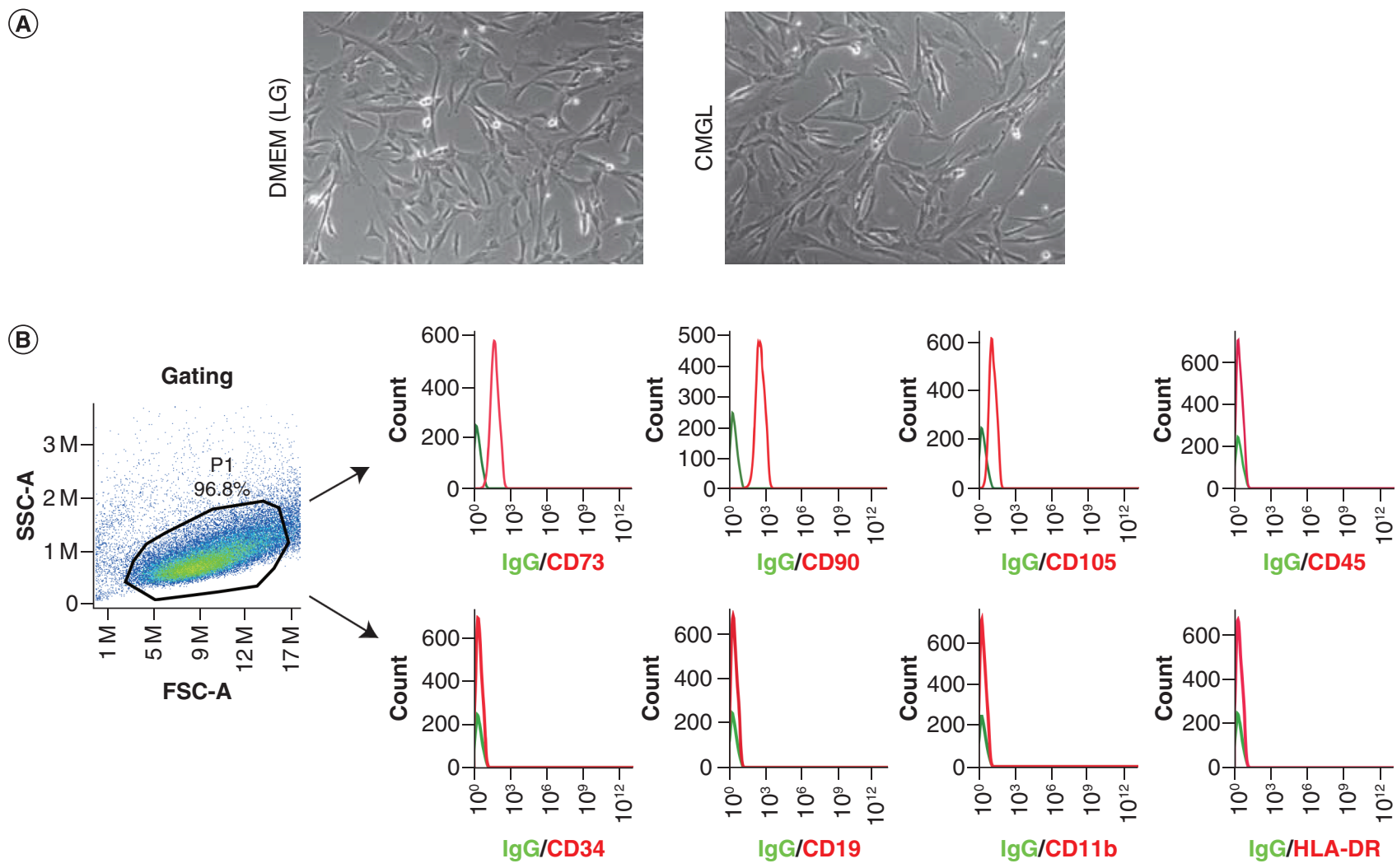

(C)

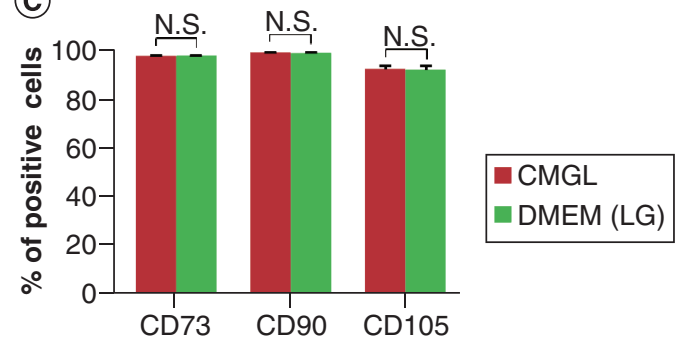

(D)

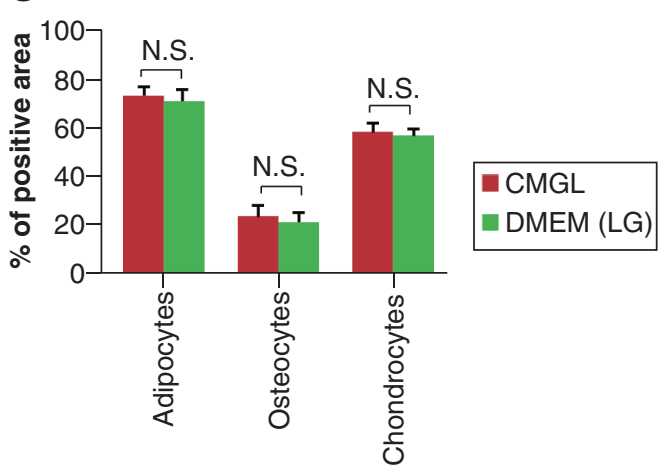

(E)

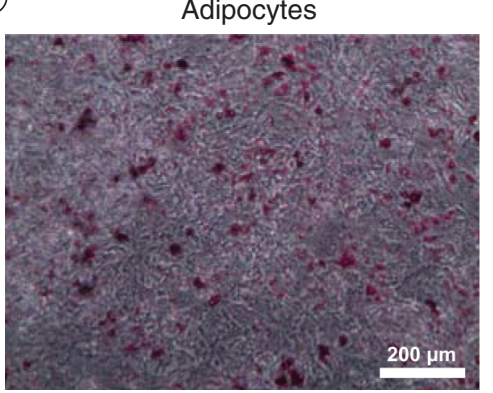

Osteocytes

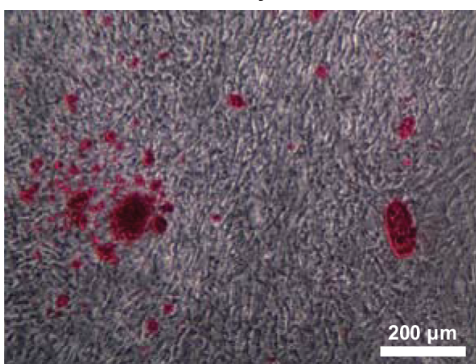

Chondrocytes

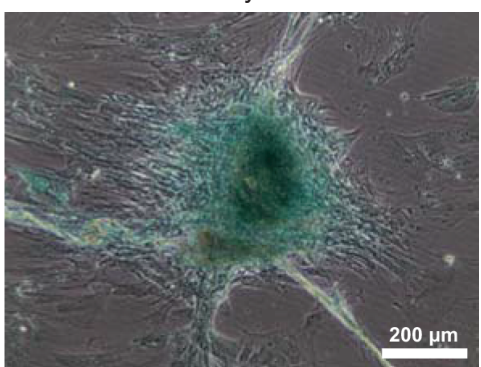

Figure 2. Characterization of mesenchymal stem cells isolated from the umbilical cord stored and dissociated with CMGL solution. (A) Cell morphology. (B) Cell surface marker analysis by flow cytometry. (C) Quantity of $C D 73^{+}, \mathrm{CD} 0^{+}$and $\mathrm{CD} 105^{+}$cell analysis by flow cytometry $(\mathrm{n}=3)$. (D) Quantity of adipocytes, osteocytes and chondrocytes after differentiation analysis by counting staining positive areas relative to total area among ten different areas $(n=3)$. (E) Representative figures for adipocytes, osteocytes and chondrocytes differentiation of MSCs.

CMGL: Our newly developed cost-effective storage solution containing calcium, magnesium, glucose-low concentration in PBS; MSC: Mesenchymal stem cell. 


\begin{tabular}{|c|c|c|}
\hline Components & CMGL (mg/l) & DMEM (LG) (mg/l) \\
\hline $\mathrm{CaCl}_{2}$ & 200 & 200 \\
\hline $\mathrm{MgSO} 4-7 \mathrm{H}_{2} \mathrm{O}$ & - & 200 \\
\hline Glucose & 1000 & 1000 \\
\hline $\mathrm{KH}_{2} \mathrm{PO}_{4}$ & 210 & - \\
\hline $\mathrm{NaH}_{2} \mathrm{PO}_{4}-2 \mathrm{H}_{2} \mathrm{O}$ & - & 141 \\
\hline $\mathrm{NaCl}$ & 9000 & 6400 \\
\hline Glycine & - & 30 \\
\hline L-Alanyl-glutamine & - & 862 \\
\hline L-Arginine hydrochloride & - & 84 \\
\hline L-Leucine & - & 105 \\
\hline L-Lysine hydrochloride & - & 146 \\
\hline L-Methionine & - & 30 \\
\hline L-Phenylalanine & - & 66 \\
\hline L-Serine & - & 42 \\
\hline L-Threonine & - & 95 \\
\hline L-Tryptophan & - & 16 \\
\hline L-Tyrosine & - & 72 \\
\hline L-Valine & - & 94 \\
\hline Choline chloride & - & 4 \\
\hline i-Inositol & - & 7.2 \\
\hline Ferric nitrate & - & 0.1 \\
\hline Potassium chloride & - & 400 \\
\hline Sodium bicarbonate & - & 3700 \\
\hline Sodium pyruvate & - & 110 \\
\hline Phenol red & - & 15 \\
\hline Cost/500 ml (RMB) & 16 & 280 \\
\hline
\end{tabular}

The formulation of DMEM (LG) was obtained from the website of Thermo Scientific company (cat. no. 10567014). The cost was evaluated based on the products made in China, which may vary among different suppliers.

CMGL: Our newly developed cost-effective storage solution containing calcium, magnesium, glucose-low concentration in PBS; LG: Low glucose (1 g/l).

for UC storage and MSC isolation, comparable to the DMEM with low glucose (Figure $1 \mathrm{~A})$. Interestingly, the long period of storage in CMGL increased the isolation efficiency of MSCs from the UC (Figure 1A). One potential explanation is that the CMGL might support the proliferation of resident MSCs in the UC. Indeed, the UC matrix contains plenty of growth factors [22], and MSCs are more proliferative at a low concentration $(1 \mathrm{~g} / \mathrm{l})$ than at a high concentration of glucose $(4.5 \mathrm{~g} / \mathrm{l})$ [23]. Therefore, CMGL might also be suitable for application in the procedure of cell isolation in addition to UC storage. Normally, to provide efficient nutrients for the isolated MSCs during the isolation procedure, DMEM was used to dilute the dissociation enzyme. The cost would be further reduced if DMEM was replaced by CMGL. Thus, the efficiency of MSC isolation in two dissociation solutions prepared with DMEM or CMGL was compared. Data showed that there was no significant difference between these two dissociation solutions, indicating that CMGL could be used to dilute the dissociation enzyme during the MSC isolation procedure. In summary, CMGL could be used for UC storage and MSC isolation with high efficient MSC isolation. 
To further demonstrate that cells isolated with CMGL-based dissociation solution and storage solution for UC delivery have classical characteristics of MSCs, the isolated cells were subjected to morphological cell surface markers and differentiation analysis. Data showed that the MSCs isolated with CMGL-based solutions had fibroblast-like morphology; were positive for CD73, CD90 and CD105; negative for CD45, CD34, CD19, CD11b and HLADR; and capable of differentiating into adipocytes, osteocytes and chondrocytes, with similar levels as MSCs isolated with DMEM/low glucose (Figure 2A-E).

\section{Author contributions}

B Lai and $\mathrm{J}$ Huang optimized the formulation and performed the MSC isolation. J Chen and W Li collected the samples. $\mathrm{H}$ Wu characterized the MSCs. J Xu designed and supervised the project, obtained financial support and wrote the manuscript.

\section{Financial \& competing interests disclosure}

This work was supported by the Natural Science Foundation of Shenzhen (grant numbers JCYJ20180305163407913, KQJSCX20180328093434771 and JCYJ20170818093720089), and the Medical Foundation of Guangdong (grant number A2018308). The authors have no other relevant affiliations or financial involvement with any organization or entity with a financial interest in or financial conflict with the subject matter or materials discussed in the manuscript apart from those disclosed.

No writing assistance was utilized in the production of this manuscript.

\section{Ethical conduct of research}

This study was approved by the ethics committee of Shenzhen University and followed the tenets of the Declaration of Helsinki. Informed consent to participate in the study was obtained from participants.

\section{Data availability}

All related data are available under request.

\section{Open access}

This work is licensed under the Attribution-NonCommercial-NoDerivatives 4.0 Unported License. To view a copy of this license, visit http: //creativecommons.org/licenses/by-nc-nd/4.0/

\section{References}

1. Peng $Y$, Chen $X$, Liu Q et al. Mesenchymal stromal cells infusions improve refractory chronic graft versus host disease through an increase of CD5+ regulatory B cells producing interleukin 10. Leukemia 29(3), 636-646 (2015).

2. Xu JY, Lee YK, Ran X et al. Generation of induced cardiospheres via reprogramming of skin fibroblasts for myocardial regeneration. Stem Cells 34(11), 2693-2706 (2016).

3. Jurewicz M, Yang S, Augello A et al. Congenic mesenchymal stem cell therapy reverses hyperglycemia in experimental Type 1 diabetes. Diabetes 59(12), 3139-3147 (2010).

4. Cipriani $P$, Carubbi F, Liakouli V et al. Stem cells in autoimmune diseases: implications for pathogenesis and future trends in therapy. Autoimmun. Rev. 12(7), 709-716 (2013).

5. Xu J, Chen J, Li W et al. Additive therapeutic effects of mesenchymal stem cells and IL-37 for systemic lupus erythematosus. J. Am. Soc. Nephrol. doi:10.1681/ASN.2019050545 (2019).

6. Xu J. Therapeutic applications of mesenchymal stem cells for systemic lupus erythematosus. Adv. Exp. Med. Biol. 1089, 73-85 (2018).

7. Shi Y, Wang Y, Li Q et al. Immunoregulatory mechanisms of mesenchymal stem and stromal cells in inflammatory diseases. Nat. Rev. Nephrol. 14(8), 493-507 (2018).

8. Wang Y, Chen X, Cao W, Shi Y. Plasticity of mesenchymal stem cells in immunomodulation: pathological and therapeutic implications. Nat. Immunol. 15(11), 1009-1016 (2014).

9. Frenette PS, Pinho S, Lucas D, Scheiermann C. Mesenchymal stem cell: keystone of the hematopoietic stem cell niche and a stepping-stone for regenerative medicine. Annu. Rev. Immunol. 31, 285-316 (2013).

10. Parekkadan B, Milwid JM. Mesenchymal stem cells as therapeutics. Annu. Rev. Biomed. Eng. 12, 87-117 (2010).

11. Uccelli A, Moretta L, Pistoia V. Mesenchymal stem cells in health and disease. Nat. Rev. Immunol. 8(9), 726-736 (2008).

12. Jiang W, Xu J. Immunue modulation by mesenchymal stem cells. Cell Prolif. 53(1), e12712 (2020).

13. Troyer DL, Weiss ML. Wharton's jelly-derived cells are a primitive stromal cell population. Stem Cells 26(3), 591-599 (2008).

14. Can A, Karahuseyinoglu S. Concise review: human umbilical cord stroma with regard to the source of fetus-derived stem cells. Stem Cells 25(11), 2886-2895 (2007).

15. Campagnoli C, Roberts IA, Kumar S, Bennett PR, Bellantuono I, Fisk NM. Identification of mesenchymal stem/progenitor cells in human first-trimester fetal blood, liver, and bone marrow. Blood 98(8), 2396-2402 (2001).

16. Guillot PV, Gotherstrom C, Chan J, Kurata H, Fisk NM. Human first-trimester fetal MSC express pluripotency markers and grow faster and have longer telomeres than adult MSC. Stem Cells 25(3), 646-654 (2007).

17. Karahuseyinoglu S, Cinar O, Kilic E et al. Biology of stem cells in human umbilical cord stroma: in situ and in vitro surveys. Stem Cells 25(2), 319-331 (2007).

18. Baksh D, Yao R, Tuan RS. Comparison of proliferative and multilineage differentiation potential of human mesenchymal stem cells derived from umbilical cord and bone marrow. Stem Cells 25(6), 1384-1392 (2007).

19. Liu $\mathrm{CB}$, Huang $\mathrm{H}$, Sun $\mathrm{P}$ et al. Human umbilical cord-dervived mesenchymal stromal cells improve left ventricular function, perfusion, and remodeling in a porcine model of chronic myocardial ischemia. Stem Cells Transl. Med. 5(8), 1004-1013 (2016).

20. Weiss ML, Troyer DL. Stem cells in the umbilical cord. Stem Cell Rev. 2(2), 155-162 (2006).

21. Jiang W, Lian W, Chen J et al. Rapid identification of genome-edited mesenchymal stem cell colonies via Cas9. BioTechniques 66(5), 231-234 (2019).

22. Sobolewski K, Malkowski A, Bankowski E, Jaworski S. Wharton's jelly as a reservoir of peptide growth factors. Placenta 26(10), 747-752 (2005).

23. Sotiropoulou PA, Perez SA, Salagianni M, Baxevanis CN, Papamichail M. Characterization of the optimal culture conditions for clinical scale production of human mesenchymal stem cells. Stem Cells 24(2), 462-471 (2006). 
